Article

\title{
A Variant Quorum Sensing System in Aeromonas veronii MTCC 3249
}

\section{Kamlesh Jangid $^{1}{ }^{*}$, Perunninakulath S. Parameswaran ${ }^{2}$ and Yogesh S. Shouche ${ }^{1}$}

1 Microbial Culture Collection, National Centre for Cell Science, Pune, Maharashtra 411007, India; E-Mail: yogesh@nccs.res.in

2 National Institute of Oceanography, Dr. Salim Ali Road, P.O. Box 1913, Kochi, Kerala 682018, India; E-Mail: param@nio.org

* Author to whom correspondence should be addressed; E-Mail: jangidk@ @ nccs.res.in; Tel.: +91-20-2570-8237; Fax: +91-20-2569-2259.

Received: 21 February 2012; in revised form: 17 March 2012 / Accepted: 20 March 2012 / Published: 26 March 2012

\begin{abstract}
We have investigated the quorum sensing control in Aeromonas veronii MTCC 3249, originally isolated as A. culicicola from the midgut of Culex quinquefasciatus. Based on biosensor assays, the bacterium showed constant production of multiple acyl-homoserine lactones (AHLs) with increasing cell-density. The luxRI gene homologs,

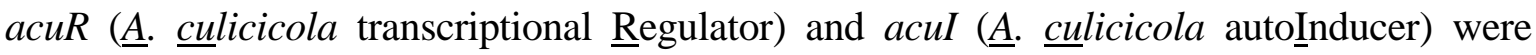
successfully amplified by inverse-PCR. Sequence analysis indicated $a c u R I$ were divergent from all known quorum sensing gene homologs in Aeromonas. Two localized regions in the C-terminal autoinducer binding domain of $a c u R$ showed indels suggesting variations in autoinducer specificity. Further, only a single copy of the quorum sensing genes was detected, suggesting a tight regulation of mechanisms under its control. Chromatography and further chemical analysis identified two AHLs in the culture supernatant: 6-carboxyHHL (homoadipyl homoserine lactone), a novel AHL, and $N$-tetradecanoylhomoserine lactone. The existence of a potentially variant quorum sensing system might therefore, reflect in some way the ecological strategies adopted by this bacterium in the mosquito midgut.
\end{abstract}

Keywords: Aeromonas; luxRI homolog; acuR; acuI; quorum sensing; AHL; mosquito midgut; 6-carboxy-HHL 


\section{Introduction}

Aeromonas spp. are important disease-causing pathogens of fish and other cold-blooded species, as well as humans [1]. Many of its virulence determinants are expressed at high cell densities in the late exponential phase and may thus be under quorum sensing control [2]. Further, the close proximity of LuxRI gene homologs to iciA, an inhibitor of chromosome replication links quorum sensing and cell division in this genus. Recently, homologs of the $V$. fischeri luxRI genes have been detected in most Aeromonas species [3], unlike a few years ago, when only two species, A. hydrophila and A. salmonicida, were known to secrete quorum sensing molecules.

The discovery of quorum sensing in Aeromonas has placed focus on this genus for the elucidation of its role in pathogenesis. Unlike A. hydrophila, wherein both the serine and metallo protease activities are under quorum sensing control [4], the general secretory pathway for the export of serine protease in A. salmonicida (exe) is not under quorum sensing control [2]. Further, the production of extracellular proteases in A. hydrophila is decreased in the presence of long chain $\mathrm{N}$-acyl-L-homoserine lactones (AHLs) such as 3-oxo- $\mathrm{C}_{12}$-HSL. Thus, the quorum sensing system could be a potential target for novel pharmaceutical agents based on the long chain AHLs to decrease the virulence of the bacterium [4].

The increasing incidences of isolation of Aeromonas species from both clinical and environmental samples have interested microbiologists worldwide. One such strain, A. culicicola MTCC 3249, later determined to be a subjective synonym of $A$. veronii [5], was isolated from the midgut of Culex quinquefasciatus by Pidiyar et al. [6]. In a study on the effect of A. veronii MTCC 3249 on the susceptibility of Culex quinquefasciatus to the Japanese encephalitis virus (JEV), the bacterium increased the susceptibility of mosquitoes to JEV when added in the mosquito's blood meal [7]. Further, 27 strains of $A$. veronii were isolated from drinking water supply in Spain [8] suggesting a diverse worldwide distribution of the species. Given that contaminated drinking water supply is a major source of diarrhoeal diseases in Spain, the pathogenic potential of this species must be tested.

In addition, gaining an insight into the quorum sensing machinery of A. veronii MTCC 3249 has become a priority. This hemolytic strain was reported to rapidly increase its cell number (2,000-fold) after the blood meal of mosquito [6]. Similar observations were reported in other species of Aeromonas that form biofilms and inhabit nutrient rich, localized environments $[2,4,9,10]$. The rapid growth of midgut bacteria after blood meal may be fuelled by the iron and protein-rich bolus of blood [11]. However, such high increase in cell number also suggests the presence of a very efficient regulatory mechanism. Given the close proximity of iciA homolog downstream of the autoinducer synthase gene in Aeromonas, the involvement of quorum sensing in increasing the cell density cannot be ruled out. This tempted us to investigate the nature of this control.

We also investigated whether $A$. veronii contained paralogs of the acuRI quorum sensing genes. We hypothesized that like many other bacteria, such as Clostridium perfringens [12], Erwinia carotovora [13], Pseudomonas aureofaciens [14], etc., A. veronii MTCC 3249 might also possess multiple quorum sensing systems. This stems from the fact that luxRI gene homologs in Aeromonas share high sequence similarity [3] and it is likely that if multiple quorum sensing systems are present in this strain, these might also share high sequence similarity with the $a c u R I$ system. Although this specific aspect has not been investigated in the genus Aeromonas, results from A. hydrophila and A. salmonicida [2,4] suggest the involvement of multiple AHLs and as-yet unidentified factors controlling different phenotypes 
under quorum sensing control. Hence, the existence of multiple quorum sensing systems cannot be ruled out. Here, we present an analysis of the quorum sensing system in A. veronii MTCC 3249 that might in some way reflect on its ecological strategies in mosquito midgut.

\section{Experimental Section}

\subsection{Bacterial Strains, Plasmids, Media, and Culture Conditions}

A. hydrophila ATCC $7066^{\mathrm{T}}$, A. veronii $\mathrm{MTCC}$ 3249, AHL responsive biosensor strain Chromobacterium violaceum CV026 [15] and E. coli JM109 harboring plasmids pSB401 and pSB403 [16] and pJBA89 [17] were maintained on Luria-Bertani (LB) medium throughout the study and incubations were carried out at $30{ }^{\circ} \mathrm{C}$. Wherever required, the medium was supplemented with ampicillin $(50 \mu \mathrm{g} / \mathrm{mL})$ or kanamycin $(40 \mu \mathrm{g} / \mathrm{mL})$ or tetracycline $(20 \mu \mathrm{g} / \mathrm{mL})$. Cell growth was monitored by measurement of the optical density of culture medium at $540 \mathrm{~nm}$.

\subsection{Growth Dependent AHL Production by A. veronii MTCC 3249}

The production of AHLs by A. veronii MTCC 3249 was detected using the recombinant plasmid pJBA89 in E. coli JM109 as described previously [3]. To determine the critical cell density at which A. veronii shows a rapid increase in the production of the AHLs, a growth phase dependent induction of bioluminescence by AHLs in sterile culture filtrate was studied as described previously [18]. Total viable count of $A$. veronii were achieved by spread plating $100 \mu \mathrm{L}$ aliquots of different dilutions in duplicates at various time intervals during the growth in $\mathrm{LB}$ at $30{ }^{\circ} \mathrm{C}$ at $150 \mathrm{rpm}$. For bioluminescence induction, $100 \mu \mathrm{L}$ supernatant fractions collected at various time intervals upon centrifugation of culture for $3 \mathrm{~min}$ at $12,000 \mathrm{rpm}$ was mixed in a 96-well plate with $100 \mu \mathrm{L}$ of the recombinant E. coli JM109 containing pSB401 diluted to an optical density of 0.8 at $450 \mathrm{~nm}$ and incubated for $30 \mathrm{~min}$. Bioluminescence counts were taken on Top Count NXT, a microplate scintillation and luminescence counter (Packard, Germany). Fold induction was calculated by substracting the counts of negative control (LB with E. coli pSB401) from the entire test samples and then dividing each sample value with the zero minute count. The positive control included $1 \mu \mathrm{M}$ final concentration of HHL. All experiments were repeated twice and final readings are an average of the two.

\subsection{DNA Isolation, PCR/Inverse PCR Amplification, Hybridization and Sequencing}

Genomic DNA was isolated from the two Aeromonas strains using the standard phenol/chloroform/ isoamyl alcohol method [19]. To PCR amplify the luxRI gene homologs from A. veronii, primers were designed using GeneRunner v3.01 (www.generunner.net) based on A. hydrophila (X89469) and A. salmonicida (U65741) luxRI gene homolog sequences. All primers were numbered according to the $3^{\prime}$ end binding site on the ahyRI gene (Table 1). PCR conditions used were: initial denaturation at $95{ }^{\circ} \mathrm{C}$ for $3 \mathrm{~min} ; 35$ cycles of denaturation at $95{ }^{\circ} \mathrm{C}$ for $1 \mathrm{~min}$, annealing at $50{ }^{\circ} \mathrm{C}$ for $1 \mathrm{~min}$ and extension at $72{ }^{\circ} \mathrm{C}$ for $1 \mathrm{~min}$; and a final extension at $72{ }^{\circ} \mathrm{C}$ for $10 \mathrm{~min}$. PCR amplicons were purified by $\mathrm{PEG} / \mathrm{NaCl}$ precipitation and sequenced in-house on an ABI-3730 automated DNA analyzer.

For Inverse PCR amplification of the flanking regions, the strategy depicted in Figure 1 was used. All the enzymatic manipulations were carried out as described previously [19] or wherever necessary 
as described by the manufacturer. Briefly, $10 \mu \mathrm{g}$ of $A$. veronii genomic DNA was digested with $10 \mathrm{U}$ of the restriction enzyme in $1 \mathrm{X}$ digestion buffer in $25 \mu \mathrm{L}$ reaction volume at $37^{\circ} \mathrm{C}$ for $12-14 \mathrm{~h}$. Upon digestion, heat denaturation was done at $80{ }^{\circ} \mathrm{C}$ for $15 \mathrm{~min}$ and $2 \mu \mathrm{L}$ of the digested fragments was checked on $0.8 \%$ agarose in $0.5 \times$ TBE. The remaining digested product was purified using the QIAQuick PCR purification kit (QIAGEN) according to manufacturer's instructions.

Table 1. List of primers used in this study.

\begin{tabular}{lll}
\hline Primer Name & Primer Sequence $\left(5^{\prime}\right.$ to $\left.3^{\prime}\right)$ & Remarks \\
\hline QS-158R & CGC ATT TTC CGC CGT CTC GG & Inverse Sequencing \\
QS-722F & GGG GGC TAG CAT ATG AAA CAA GAC C & Degenerate \\
QS-1444R & TTA TTG CAT CAG CTT GGG GAA GTT G & Degenerate \\
QS-1469F & CAC CAA CTT CCC TAA ACT GAT GCA ATA G & Inverse Sequencing \\
QSAcuR-255R & GGT TCC AGT AGA TGG GCA GCG TC & Inverse \\
QSAcuR-525F & GGT TGT GCG CCT GAG GGA TTC G & Inverse Probe \\
AcuIF & ATG TTG GTT TTC AAA GGA AAA TTG & A. veronii specific \\
AcuIR & TTA TAT CTG GGC CGC TAA CTC ATG GGA & A. veronii specific \\
AcuRF & ATG AAA CAA GAG CAA CTG TTT GAG TAT & A. veronii specific \\
AcuRR & CTA TTG CAT CAG TTT AGG GAA GTT GGT & A. veronii specific \\
\hline
\end{tabular}

Figure 1. Strategy for Inverse PCR amplification of acuRI from A. veronii.

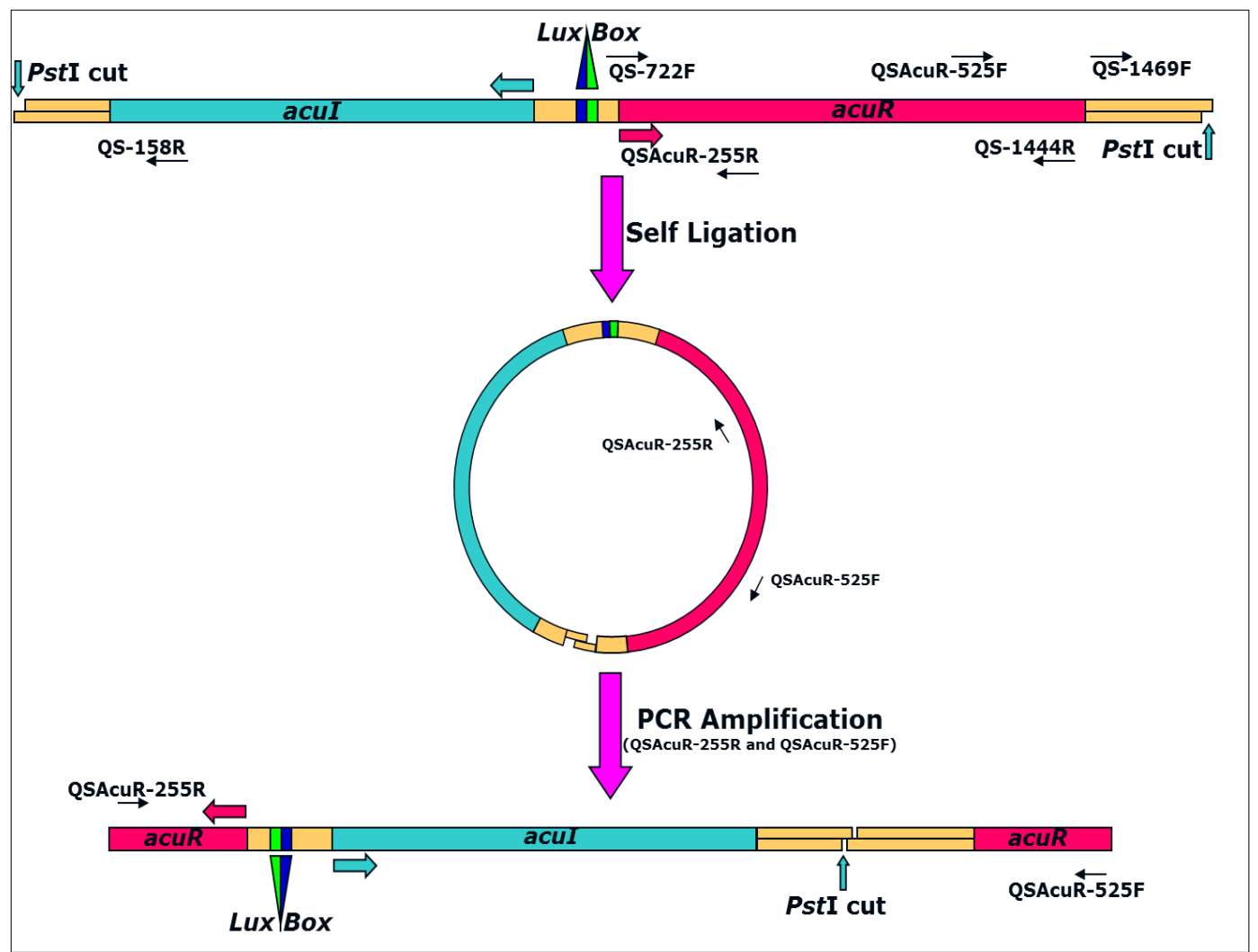

Purified DNA fragments were self-ligated in $10 \mu \mathrm{L}$ reaction volume containing $5 \mu \mathrm{L}$ of purified product, $1 \times$ ligation buffer and $20 \mathrm{U}$ of the T4 DNA ligase at $16{ }^{\circ} \mathrm{C}$ for $16 \mathrm{~h}$. Upon ligation, $2 \mu \mathrm{L}$ was used for a $50 \mu \mathrm{L}$ PCR reaction using primers QSAcuR-255R and QSAcuR-525F under similar conditions as described above except that annealing was at $60{ }^{\circ} \mathrm{C}$ and cycle elongation was at $72{ }^{\circ} \mathrm{C}$. Amplicons 
$(10 \mu \mathrm{L})$ were run against $1 \mathrm{~kb}$ plus DNA marker (Invitrogen, USA) in 1\% agarose gel in $1 \times$ TBE buffer at $5 \mathrm{~V} / \mathrm{cm}$ for $4 \mathrm{~h}$ and then processed for southern hybridization to target the fragment containing the gene of interest. All protocols for blot preparation, probing, hybridization and developing were carried out essentially as described previously [19]. Blots were prepared on Hybond $\mathrm{N}^{+}$paper (Amersham Pharmacia, USA) after depurination, denaturation and neutralization treatments followed by $\sim 4 \mathrm{~h}$ transfer in 20x SSC using vacuum blotting apparatus. Upon transfer, the blot was washed gently in $2 \times$ SSC, air dried and processed for hybridization at $55^{\circ} \mathrm{C}$ as described previously [5]. PCR product amplified using primers QSAcuR-525F and QS-1444R was used as the probe.

DNA fragments corresponding to the putative acuRI genes detected in Southern hybridization were eluted from the remaining $40 \mu \mathrm{L}$ amplicons run on $1 \%$ agarose gel in $1 \times$ TAE $(\mathrm{pH}=8.0)$ under similar conditions. Elution was carried out using the QIAquick gel extraction kit (QIAGEN) according to manufacturer's instructions. The eluted fragments were cloned in pGEM-T Easy vector (Promega, USA) at molar ratios of 1:3 for vector:insert in a final reaction volume of $5 \mu \mathrm{L}$ and transformed in $E$. coli JM109 competent cells as described previously [20]. LB Agar plates supplemented with ampicillin $(100 \mu \mathrm{g} / \mathrm{mL}), \mathrm{X}$-gal $(40 \mu \mathrm{g} / \mathrm{mL})$ and IPTG $(0.1 \mathrm{mM})$ were used for plating $100 \mu \mathrm{L}$ transformed culture suspension. Clones were picked after $12-14 \mathrm{~h}$ incubation at $37^{\circ} \mathrm{C}$ and screened by direct-colony PCR using vector specific PUC1 and PUC2 primers under the conditions described above for inverse PCR. Amplicons from positive clones were purified using $\mathrm{PEG} / \mathrm{NaCl}$ precipitation and sequenced as above. Nucleotide sequence of the complete genes along with its flanking regions is submitted to GenBank with accession number AY989817.

\subsection{Determination of acuRI Copy Number}

Southern hybridization for acuRI copy number was performed as described previously for $r r n$ operon [21]. PCR amplified $a c u R$ gene from A. veronii using primers $A c u R F$ and AcuRR was used as the probe. Amplicons were purified by PEG-NaCl precipitation, and random labelled using Megaprime DNA labelling system (Amersham Pharmacia Biotech UK Ltd.). The PCR conditions used were as described above except that annealing was at $55^{\circ} \mathrm{C}$. Hybridization was performed at $60{ }^{\circ} \mathrm{C}$ for $14 \mathrm{~h}$ in a solution containing $5 \times \mathrm{SSC}, 0.5 \% \mathrm{SDS}, 5 \times$ Denhardt's solution, $0.2 \mathrm{mg}$ of denatured salmon sperm DNA $\mathrm{mL}^{-1}$ and $2 \mathrm{ng}$ of radiolabeled probe $/ \mathrm{mL}$ at a specific activity of $>1 \times 10^{8} \mathrm{dpm} / \mu \mathrm{g}$.

\subsection{Chemical Characterization of AHLs Produced by A. veronii MTCC 3249}

For thin-layer chromatography (TLC) detection of AHLs, $5 \mathrm{~mL}$ of A. veronii culture supernatant was extracted three times with dichloromethane (7:3 supernatant/dichloromethane). The dried extract was reconstituted in $50 \mu \mathrm{L}$ HPLC grade acetonitrile and $5 \mu \mathrm{L}$ samples were subjected to analytical TLC on $\mathrm{C}_{18}$ reverse-phase chromatography plates (catalogue no. 4801425 ; Whatman), using 60\% (vol/vol) methanol in water as the mobile phase as described previously [22]. AHLs were identified by overlaying the chromatograms with a thin layer of LB agar $(45 \mathrm{~mL})$ seeded with CV026 (5 mL overnight culture). Plates were incubated at $30{ }^{\circ} \mathrm{C}$ overnight and examined for purple spots. Synthetic AHL standards applied in $2 \mu \mathrm{L}$ volume onto the plates as a common mixture of BHL (catalogue no. 09945; Fluka), HHL (catalogue no. 09926; Fluka), OHL (catalogue no. 10940; Fluka), DHL (catalogue no. 17248; Fluka) and dDHL (catalogue no. 17247; Fluka) were used for reference. 
Putative AHLs were extracted and purified from $12 \mathrm{~L}$ of stationary-phase A. veronii culture grown in M9 medium (SIGMA, USA) with 0.2\% acid hydrolyzed casein (Oxoid) as described previously [2]. Extracts were vaccum dried on a Büchi rotavapor R-200 (Büchi Labortechnik, Switzerland), reconstituted in acetonitrile, and then subjected to analytical TLC (as above) and preparative HPLC (as following). Fractions were separated using Supelco PLC8 (250 by $21.2 \mathrm{~mm}$ ) column (Chromeleon, DIONEX Corporation, USA) with an isocratic mobile phase of $70 \%$ (vol/vol) acetonitrile in water at a flow rate of $2 \mathrm{~mL}$ per min and monitored at $210 \mathrm{~nm}$. Fractions showing activity in the CV026 reporter assay were pooled and re-chromatographed by using $60 \%$ (vol/vol) acetonitrile in water; the procedure was repeated, using a final chromatographic separation employing 30\% (vol/vol) acetonitrile in water. Active fractions with same retention times were pooled and analyzed by MS and NMR.

\section{Results and Discussion}

\subsection{Production of AHLs by A. veronii MTCC 3249}

Sensitive detection of AHLs secreted by A. veronii was achieved by using recombinant derivatives of $E$. coli containing genes expressed only in the presence of AHLs and measured quantitatively. While A. veronii MTCC 3249 has already been shown to possess LuxRI gene homologs [3], the bioassay based detection reconfirmed the corresponding phenotype in this strain (Figure 2). The classical "T" shows the decreased $g f p$ expression by the indicator strain as a function of the diffusion of the compound in the medium. The production of AHLs was highly correlated with increasing cell density of A. veronii (Figure 3(a)). A curve with zero slope was observed when fold induction/OD540 was plotted (Figure 3(b)) indicating that the production of AHLs was constant and proportional to $\mathrm{CFU} / \mathrm{mL}$ of $A$. veronii MTCC 3249, and there was a linear correlation between AHL amounts and production of light by $E$. coli JM109 with pSB401. The slight differences could be minor variations due to the experimental error of measurements.

Figure 2. Expression of $g f p$ by E. coli JM109 pJBA89 in response to AHL production by A. veronii MTCC 3249.

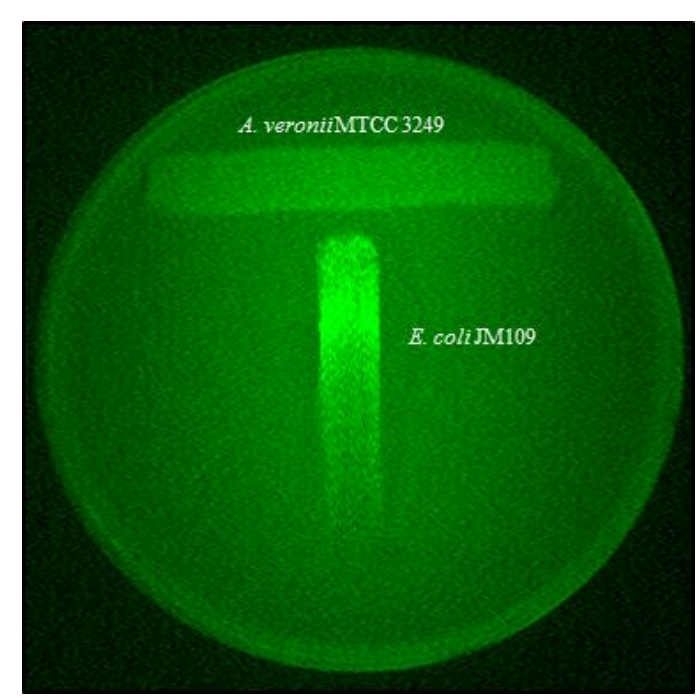


Figure 3. Growth dependent AHL production by A. veronii MTCC 3249. (a) Fold induction of bioluminescence in E. coli JM109 with pSB401 as a function of increasing AHL concentration in the culture supernatant; (b) Constant production of AHLs by A. veronii in the culture supernatant proportional to its $\mathrm{CFU} / \mathrm{mL}$.
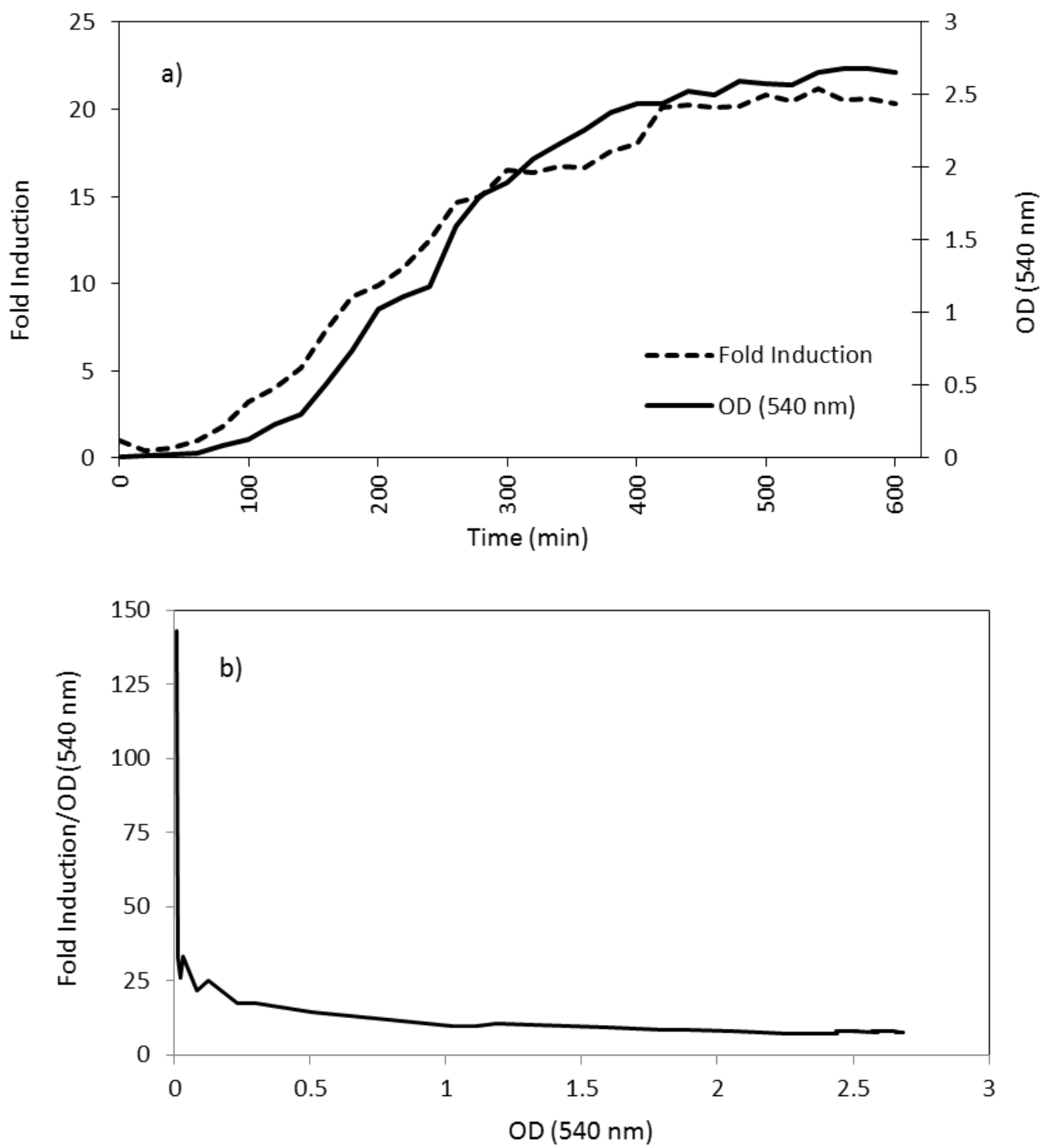

\subsection{A. veronii MTCC 3249 acuRI System}

Based on the sequence analysis of $a h y R I$ and $a s a R I$, eight primer pairs were designed to amplify the corresponding LuxRI gene homologs from A. veronii. However, only one pair, QS-722F and QS-1444R amplified a single DNA fragment ( $790 \mathrm{bp})$, determined to be the homolog of LuxR-type

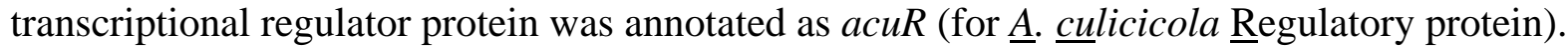

The flanking regions of the $a c u R$ gene were amplified by Inverse PCR (Figure 1). Of the four restriction enzymes: BglII, EcoRI, HindIII and PstI, chosen on the basis of absence of recognition sites in both $a c u R$ and ahyRI/asaRI sequences, only PstI digest of A. veronii MTCC 3249 yielded a single band. The region corresponding to luxI homolog was annotated as acuI (for $\underline{A}$. $\underline{\text { culicicola autoInducer }}$ synthase protein). 
Both $a c u R$ and acuI shared very low sequence similarity with previously known luxRI homologs in Aeromonas. Thus, acuR shared only $85-87 \%$ sequence similarity with ahyR and asaR, whereas the latter two shared 99\% sequence similarity between them. Similarly, acuI shared only 70-71\% sequence similarity with ahyI and $a s a I$, whereas the latter two were $88 \%$ similar. This low similarity probably explains the failure of attempts to get the acuI gene fragment using degenerate PCR and heterologous southern hybridization.

In addition to the low similarity, the $a c u R I$ gene sequence showed unique features suggesting a variant quorum sensing system in A. veronii. Specifically, a $6 \mathrm{nt}$ deletion in acuR (position 1225), an insertion in acuI (position 32), two separate deletions in acuI (positions 48 and 61) and a shorter lux box region were present in the $a c u R I$ gene sequence (Figure 4). These indels were important because the insertion lied in the primer binding site of the degenerate primers and justified the inability to PCR amplify the $a c u I$ fragment in the first step. Further analysis of $a c u R$ sequence at NCBI's Conserved Domain Search (CD Search) confirmed that the $6 \mathrm{nt}$ deletion lied in the C-terminal binding domain of the general family of transcriptional regulators. This domain contains a helix-turn-helix motif and binds DNA and thus, the deletion may be important, although this has not been investigated yet.

Figure 4. luxRI homolog sequences of A. hydrophila (ahyRI, X89469), A. salmonicida (asaRI, U65741), and A. veronii (acuRI, AY989817). luxI homologs (red), luxR homologs (green), and ygg homolog (purple). Region of dyad symmetry, $><$, not homologous to the lux box consensus sequence (blue region). Pst I site (underlined).

asaRI
ahyRI
acuRI

asaRI
ahyRI
acuRI

asaRI
ahyRI
acuRI

asaRI
ahyRI
acuRI

asaRI
ahyRI
acuRI
asurar
a
ahy
acuRI
acuRI

CCCGGGATTTTTT-TATGTGG--CGTTTACTC-GGTGACCAGTTCCCTGGCATCGGCATA 56 CCCGGGATTTTTT-TGCGTCG--CCTTTATTC-TGTGACCAGTTCGCGCGCCTCGTCATA 56

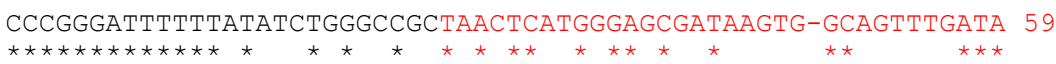

TTCGCCCTGCATG-GGGTGGCCGACGGCACGGGCGAAACGTTCATCCAGATGAAAGCGGA 115 GGCACCCTGCAGG-GGCTGGCCGACGGCACGGGCGAACCGCTCATCAAGATGGAAGCGGA 115 GT-GTCCGATAAGTGGCTGCGAGACCGCCAGGGCAAATCGCTCATCCAGATAAAAGCGCT 118

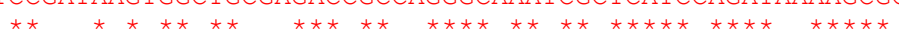

TTCCGACCCCGCGCACGGCGCCCAGATCCACGGCTTGACGGTGACCGAGTCGCTCTATGG 175 TCCCCACCCCGCGCACGGCGCCCAGATCCACCGCCTGGCGGTGACCGAGCCGTTCGATGG 175 GGCCGACACCTTTCACTGCACCGAGATCTACCGCTTGGCGGTGGCCCAGACGAGCCATAG 178

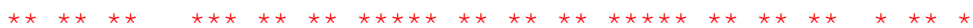

GCAGGCCGAGACGGCGGAAGATACGTTCCACCGGCAGGCTGACCACGGCGACCAGTTCCC 235 GCAGACCGAGGCGGCGGAAGATCCGCTCTACCGGCAGGCTGACCACGGCAACCAGCTCTC 235 GGATTCCGAGACGGCGGAAAATGCGTTCTGCGGGCAGGCTGGCTACACCAACAAACTCTT 238

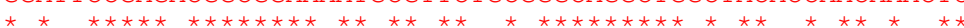

GGATCCCCTTCGCCCTGGCGAAGGCATAAACCTCGCGGAAGATGACGCAGGTCAGCTCGC 295 GGATCCCCTGCGCCTTGGCGAAGGCATAGACCTCGCGGAAGATGATGCAGGTCAGTTCGC 295 TGATGCCGTTGGCTGTAGCAAACGCATAGGCTTCCCGAAGGAGTACGCAGGTCAGCTCGC 298

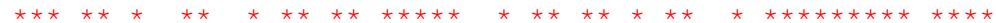
TGATGCCGTTGCCGAGCCGGGGAGCCCGTTCGGCATCGATGGCGAGGCGGGTCAGCTCCC 355 TGACACCATTGGTCATGCACGGAGCGCGGGTCGCATCGATAGCCAGACGAGTGAGTTCCC 358

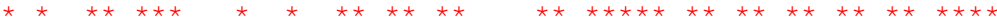




\section{Figure 4. Cont.}

asaRI ahyRI acuRI

CGATCAGCACCCAATGGGTGTCCGGGGTGTCGAAGCTGTCCTGCTCCAGACCACGGTGAG 535 CGATCAGCACCCAGTGGGTATCAGGGGTATCGAAACTGTCCTGCTCCAGACCACGGTGGG 535 CGACCAGTACCCAATGGGTGTCTGGCGTGTCAAAGCGGTCGCGTTCCAAACCGTGGTAAG 538

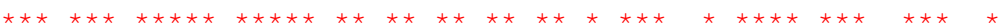

ACTCCACATCCCAGCCGAGACGATCGGAGAAGACGCGATTACGGAAGCGATAAAGCTCGT 595 ATTCCACATCCCAGCCGAGGCGATCGGAGAAGACGCGATTGCGAAAGCGATAAAGCTCGT 595 ACTCGACGTCCCAGCCTAGCCGGTCGGAAAAGACGCGATTTCGAAATTGATAGAGTTCGT 598

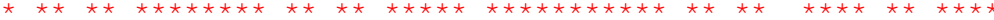

TTTCTACCTCCCATCTGGGGTGTTCTTTTAATTTCCTTTGAAAACAAGCATTCAGATGT 655 TTTCTACCTCCCATCTGGGGTGTTCTTTTAATTTTCCTTTGAAAACAAGCATTCAGATGT 655 TCTCTACGGCGTTTCGTGGGTGATCTCTCAATTTTCCTTGAAAACCAACATTATGAACT 658

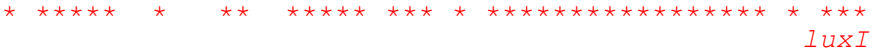

CTCCATTTCAGTGTTCGGCGCTATAATCGAGGCCCTGGCCAGGGGGGCTAGCATATGAAA 715 CTCCATTTCAGTGTTCGGCGCTATAATCGAGGCCCTGGCCAGGGGGGCTAGCATATGAAA 715 CTCTCTCTGCCTGTTCGCCGTTATAATCAGAAGCTTGGCCAGGGGGGCTAGCATATGAAA 718

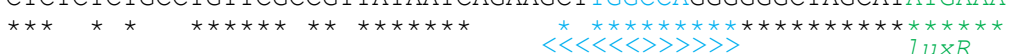

CAAGACCAACTGCTTGAGTACCTCGAACATTTCACTTCGGTAACAGACGGGGATCGTTTG 775 CAAGACCAACTGCTTGAGTACCTCGAACATTTCACTTCGGTAACGGACGGGGATCGTTTG 775 CAAGAGCAACTGTTTGAGTATCTGGAACATTTCACTTCGGTGACGGATGGTGACCGTCTT 778

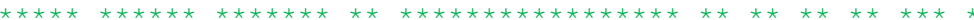

GCCGAGTTGATCGGTCGATTCACGCTCGGTATGGGCTATGACTACTACCGGTTCGCACTG 835 GCCGAGTTGATCGGTCGGTTCACGCTCGGTATGGGTTACGACTACTACCGGTTTGCGCTG 835 GCCGGATTGATCGGTCGCTTTACGGTCGATATGGGTTATGACTATTACGGCTTTACCCTG 838

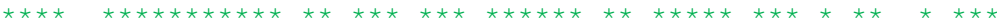

ATCATTCCCATGTCGATGCAAAGGCCAAGGTGGTGCTGTTCAATCAGTGCCCTGACTCC 895 ATCATTCCCATGTCGATGCAAAGACCCAAGGTGGTGCTGTTCAACCAGTGCCCAGACTCC 895 ATCATTCCGATGTCGATGCAAAGACCCAAAGTGTACTGTTTAATCAGTGCCCTATCTCT 898

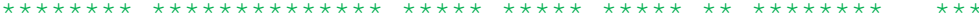

TGGGTGCAGGCCTATACGGCCAACCATATGCTGGCCTGTGATCCCATCATCCAGCTGGCC 955 TGGGTGCAGGCCTACACGGCCAACCATATGCTGGCCTGTGATCCCATCATTCAATTGGCC 955 TGGGTACAGACCTATACCGATAACAACATGCTGGCGTGCGACCCTGTGATTCAGTTGGCA 958

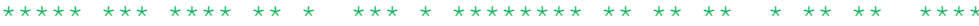

CGCAAGCAGACCTTGCCCATTTATTGGAACCGACTGGATGAACGGGCGCGTTTTCTGCAA 1015 CGCAAGCAGACCTTGCCCATTTACTGGAACCGGCTGGATGAGCGGGCGCGTTTTCTGCAA 1015 CGCAAGCAGACGCTGCCCATCTACTGGAACCGCCTCGATGAGCGGGCTCGCTTTCTGCAA 1018

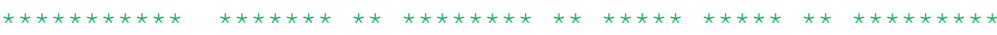

GAGGGCAGTCTGGATGTGATGGGGTTGGCGGCAGAGTTCGGGCTGCGAAACGGTATTTCG 1075 GAGGGCAGTCTTGACGTGATGGGGTTGGCGGCGGAGTTCGGGCTGCGCAACGGAATTTCG 1075 GAGGGAAGCATGGATGTGATGGGACTGGCCGCCGAGTTCGGATTGCGCAATGGCATCTCC 1078

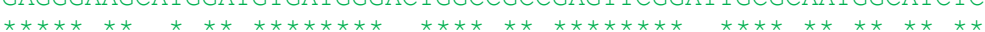

TTTCCGCTGCACGGTGCGGCGGGTGAAAACGGCATACTGTCGTTCATCACCGCCGAGCGG 1135 TTCCCGTTGCACGGTGCGGCAGGGGAGAACGGCATACTGTCGTTCATCACCGCCGAACGG 1135 TTCCCTCTGCACGGCGCAGCCGGGGAGAACGGGATCCTCTCCTTTATCACCTCCGAGCGG 1138

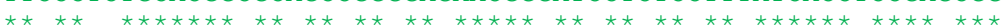

GCGTCAAGCGATCTGCTGCTGGAGTCCTCGCCCATCCTCTCCTGGATGTCCAACTACATC 1195 GCATCGAGCGATCTGCTGCTGGAGTCCTCGCCCATCCTCTCCTGGATGTCCAACTACATC 1195 GCTTCGAGCGATCTGCTGCTGGAGTCCTCGCCAATCCTCTCCTGGATGGCCAATTACATC 1198

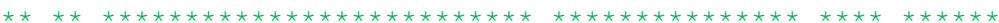

TTCGAGGCGGCGATCCGGATTGTGCGGGTCAGTCTGCGGGAAGATGACCCTCAGGAGGCC 1255 TTCGAGGCGGCGATCCGGATTGTGCGGGTCAGTCTGCGGGAAGATGACCCTCAGGAGGCC 1255 TTCGAGGCTGCGATCAGGGTTGTGCG------CCTGAGGGATTCGGATCAACAAGCCGCG 1252

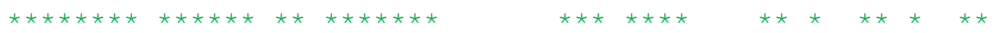


Figure 4. Cont.

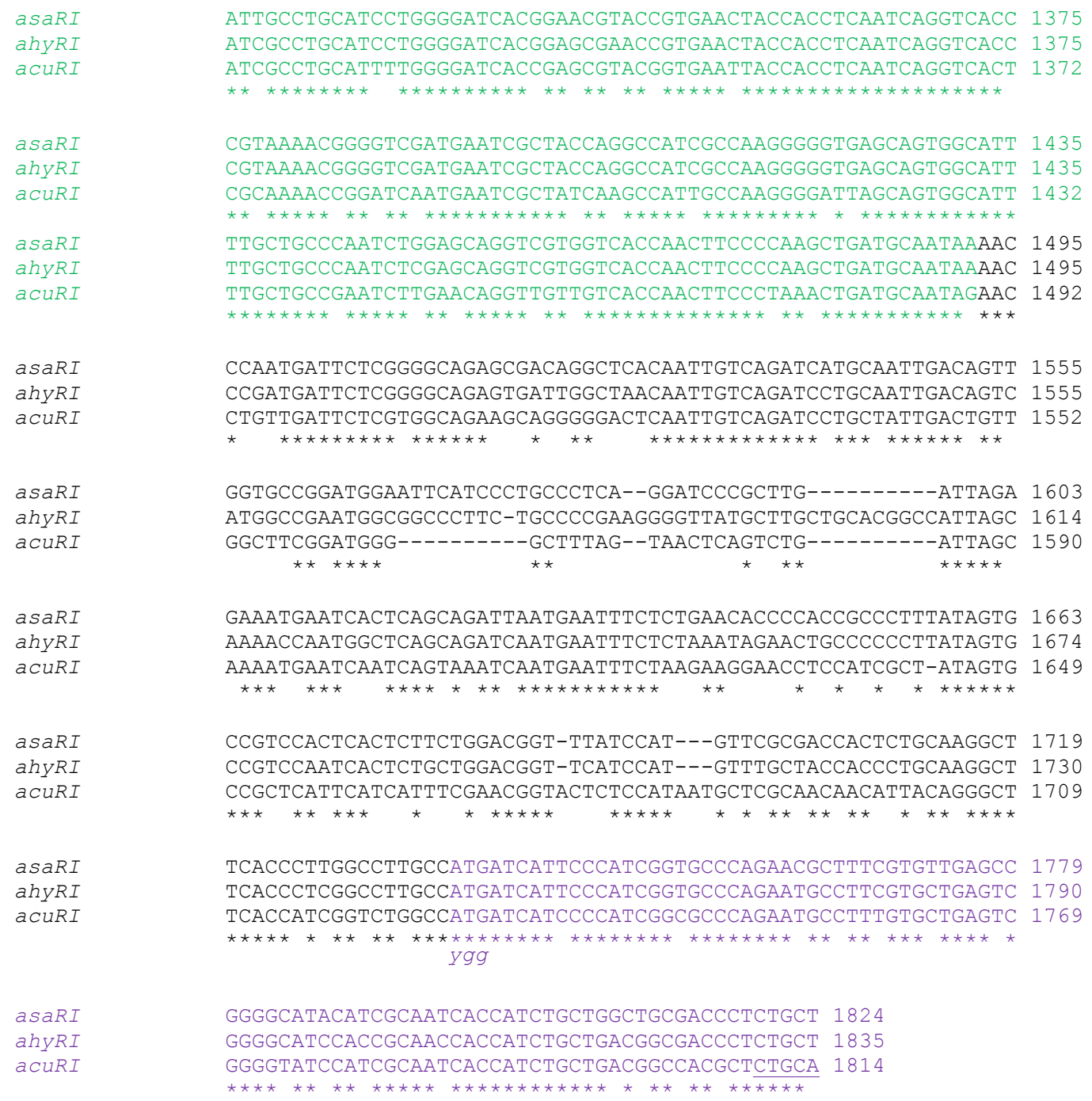

A. veronii MTCC 3249 possessed a single copy of the quorum sensing genes suggesting a tight regulation by a singular type of autoinducer (Figure 5). In order to express multiple factors under the control of a single transcriptional factor, there is always a high demand for rRNA transcription. Consistent with this, the strain also possessed 10 copies of the $r r n$ operon suggesting strong and efficient transcriptional machinery and a unique ecological strategy adopted by the organism during stages of rapid growth [21]. Given the central role of rRNAs in the regulation of ribosome synthesis, it is conceivable that the number of rRNA operons may dictate the rapidity with which microbes can synthesize ribosomes and respond to favorable changes in growth conditions [23,24]. The copy number of rRNA operons per bacterial genome, which varies from 1 to as many as 15 , therefore, reflects an ecological strategy that is characterized either by rapid response to resource input or efficient allocation of resources under constant, slow-growth environments $[25,26]$. Hence, a detailed investigation into the genes that are regulated by this system would enhance our understanding of the potentially pathogenic mechanisms under its control. 
Figure 5. acuRI copy number in A. veronii MTCC 3249 using southern hybridization. Lane 1: 1 kb Plus DNA marker (Invitrogen); lane 2: HindIII digest; and lane 3: PstI digest.

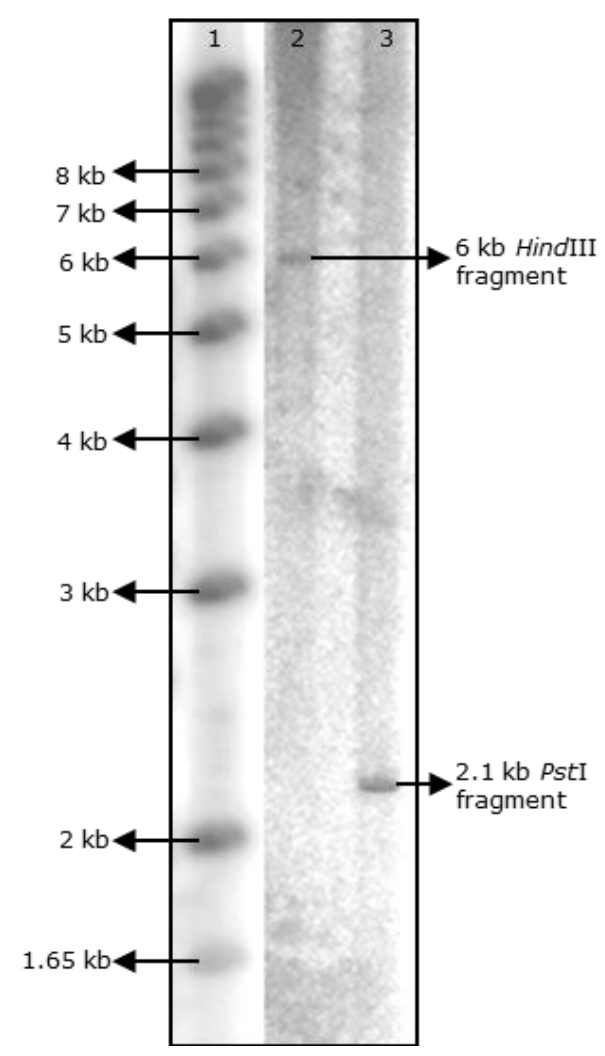

\subsection{Chemical Characterization of AHLs Produced by A. veronii MTCC 3249}

Using different biosensor strains enabled the detection of a wide range of AHLs produced by A. veronii. TLC overlay analysis with $C$. violaceum $\mathrm{CV} 026$ revealed a major AHL, which corresponded to the $R_{f}$ values obtained for synthetic HHL (Figure 6). Additionally, an unknown CV026-positive spot, probably representing a more polar compound, migrated between the synthetic HHL and OHL standards. Although this required further confirmation, our results agree to an extent with previous observations that multiple AHLs are secreted by other members in the genus Aeromonas. Studies with A. hydrophila and A. salmonicida reported two AHLs, BHL (major) and HHL (minor), secreted by the same strain and an additional chemical compound, the identity of which is not yet known [2].

The results of MS, NMR and IR with the purified extracts confirmed this observation and one of the autoinducer compounds was identified to be a carboxyl-acid side chain derivative of $N$-Heptanoyl-Lhomoserine lactone. An active HPLC fraction of the AHLs secreted by A. veronii MTCC 3249 was further analyzed on MS and NMR (for details see [27]). The IR spectrum peaks at 1754 indicated a gamma -lactone group, as is common in homoserine lactones (Figure 7). The carbonyl peaks at 1,715, $1,677,1,667$, etc., could be attributed to free carboxylic acid or amide groups. The strong absorption at 3,500 indicated $\mathrm{OH} / \mathrm{NH}$ stretching vibrations. 
Figure 6. TLC analysis of AHLs produced by A. veronii MTCC 3249. Lane 1: mixture of synthetic AHL standards, HHL and OHL; and lane 2: acetonitrile reconstituted extract of AHLs from the culture supernatant.

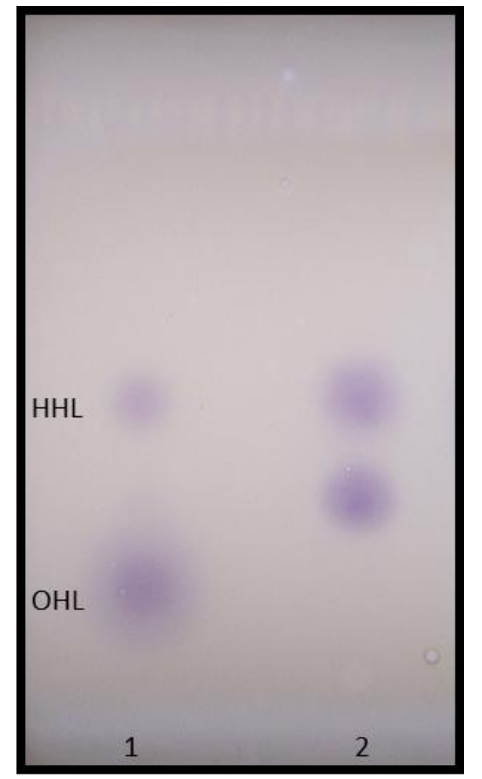

Figure 7. IR spectra of $C$. violaceum CV026 positive HPLC fraction from culture supernatant of A. veronii MTCC 3249.

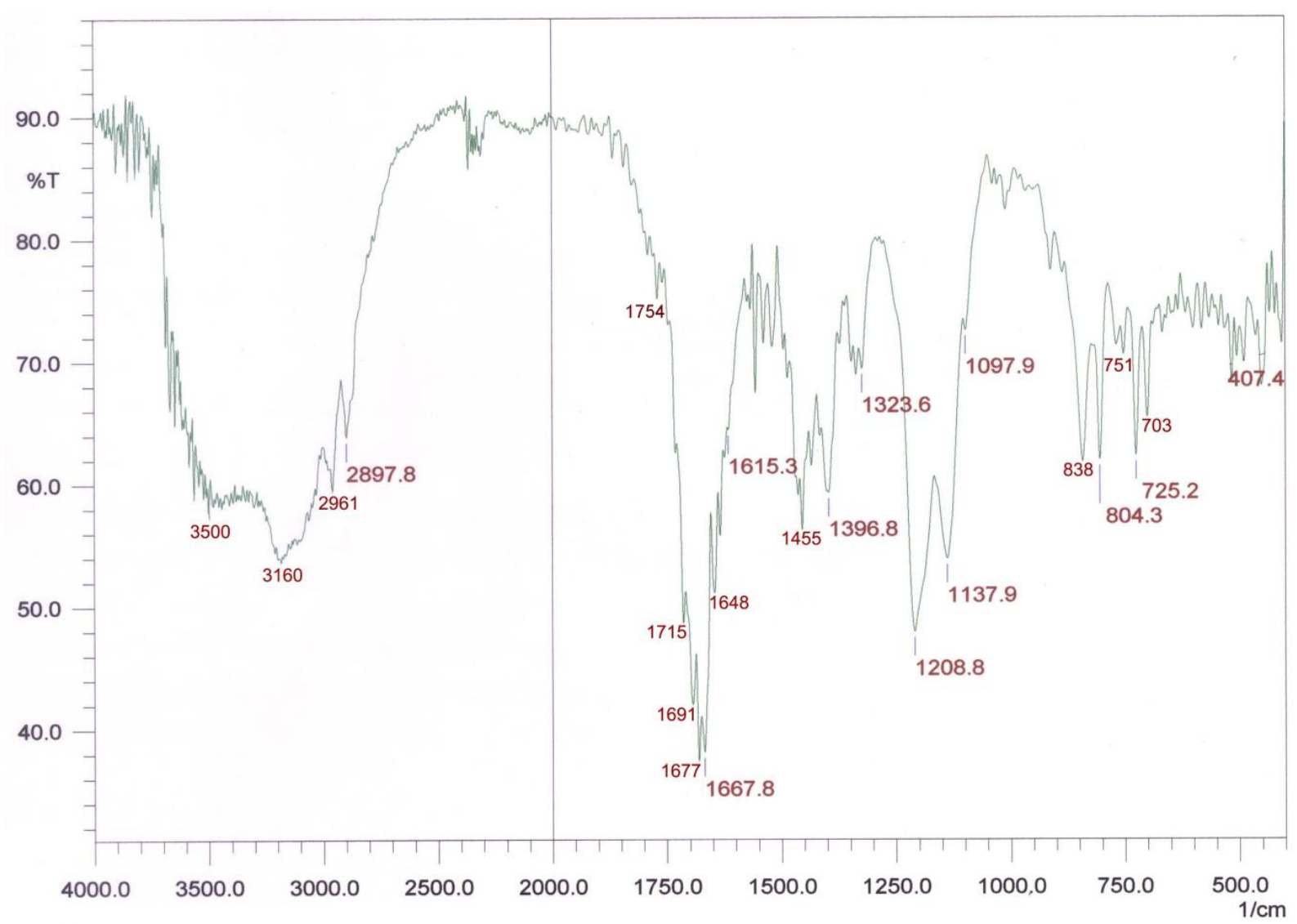

The mass spectrum indicated presence of two homoserine lactones of molecular weights 311 and 243 , respectively. Thus, the prominent peaks (Figure 8) at $m / z 645(2 \mathrm{M}+\mathrm{Na})^{+}, 623(2 \mathrm{M}+\mathrm{H})^{+}, 334$ 
$(\mathrm{M}+\mathrm{Na})^{+}, 312(\mathrm{M}+\mathrm{H})^{+}, 283\left(\mathrm{M}-\mathrm{CH}_{3} \mathrm{CH}_{2}\right)^{+}, 239(\mathrm{M}+\mathrm{H}-28-17-28)^{+}, 211(\mathrm{M}+\mathrm{H}-101)^{+}$indicated the presence of a compound of molecular weight 311 , while the values at $m / z 509(2 \mathrm{M}+\mathrm{Na})^{+}, 487$ $(2 \mathrm{M}+\mathrm{H})^{+}, 266(\mathrm{M}+\mathrm{Na})^{+}, 244(\mathrm{M}+\mathrm{H})^{+}, 222(\mathrm{M}-44+\mathrm{Na})^{+}, 200(\mathrm{M}-44+\mathrm{H})^{+}$indicated presence of a compound having 243 as the molecular weight. Given the homoserine lactone ring, the first compound has $\mathrm{C}_{14}$ acid (myrstic acid or tetradecanoic acid) as acyl group (Figure 9), corresponding to an elemental composition of $\mathrm{C}_{18} \mathrm{H}_{34} \mathrm{NO}_{3}$. The $(2 \mathrm{M}+\mathrm{H})^{+}$adduct ions of compound 1 are seen at $\mathrm{m} / z 623(2 \mathrm{M}+\mathrm{H})^{+}$ and $645(2 \mathrm{M}+\mathrm{Na})^{+}$. Other characteristic ions for compound 1 were seen at $\mathrm{m} / \mathrm{z} 283(312-29)^{+}$or $\left(\mathrm{M}+\mathrm{H}-\mathrm{C}_{2} \mathrm{H}_{5}\right)^{+}$. This loss of terminal ethyl group is known for long chain compounds, such as myrstic acid. Similarly, compound 2 has $\mathrm{C}_{7}$ dicarboxylic acid as the acyl moiety. The elemental composition of compound 2 is $\mathrm{C}_{11} \mathrm{H}_{17} \mathrm{NO}_{5}$. The observed pseudomolecular ions at $\mathrm{m} / \mathrm{z} 244$ and 266 correspond to $\mathrm{C}_{11} \mathrm{H}_{18} \mathrm{NO}_{5}(\mathrm{M}+\mathrm{H})^{+}$and $\mathrm{C}_{11} \mathrm{H}_{17} \mathrm{NO}_{5} \mathrm{Na}$, respectively. The $(2 \mathrm{M}+\mathrm{H})^{+}$adduct ions of compound 2 are seen at $\mathrm{m} / z 509(2 \mathrm{M}+\mathrm{Na})^{+}$and $487(2 \mathrm{M}+\mathrm{H})^{+}$ions. The peak at $\mathrm{m} / z 200(244-44)$ or $\left(\mathrm{M}+\mathrm{H}-\mathrm{CO}_{2}\right)$ is characteristic for carboxylic acids.

Figure 8. MS/MS scan of $C$. violaceum CV026 positive HPLC fraction from culture supernatant of $A$. veronii MTCC 3249.

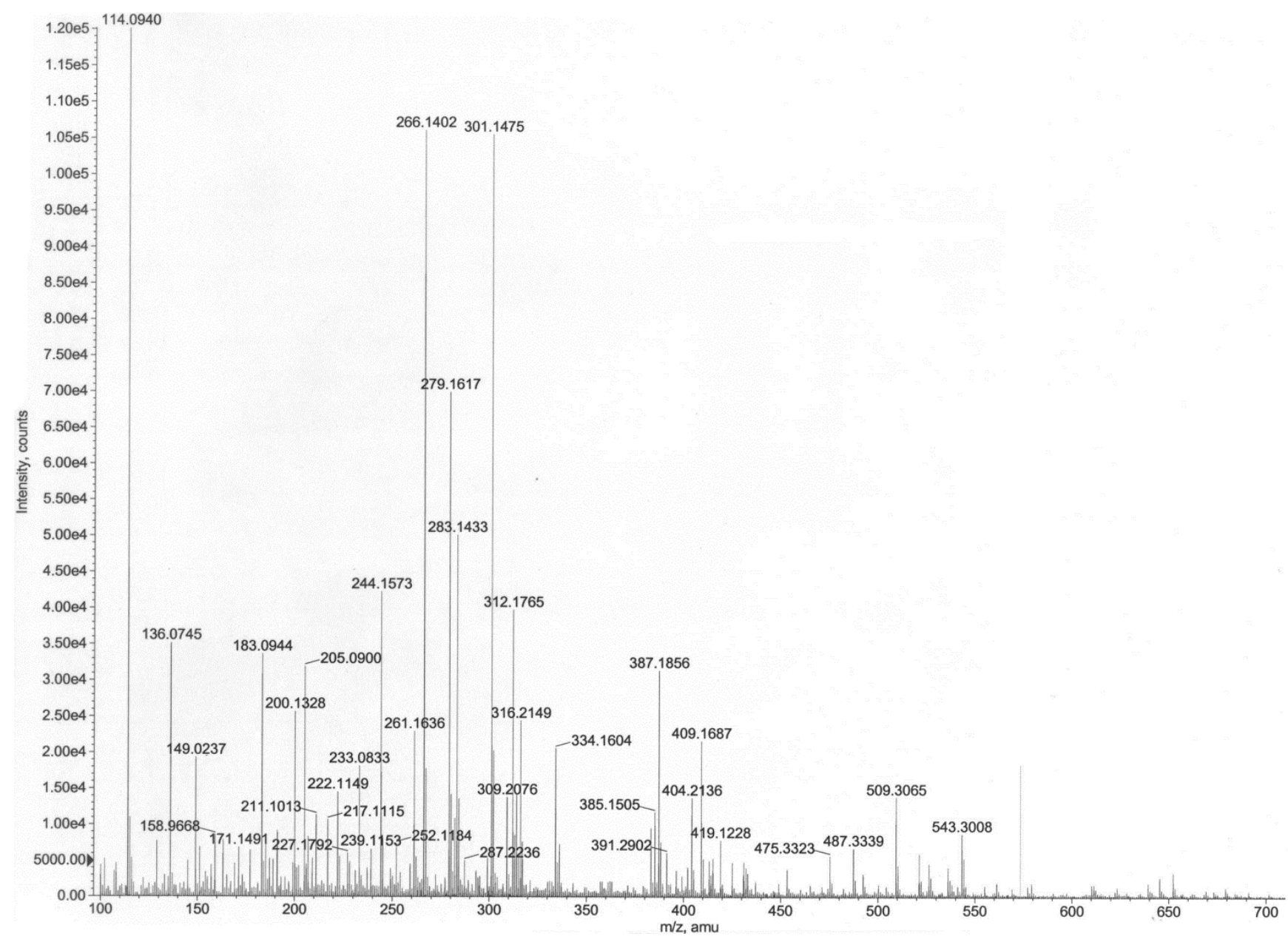


Figure 9. Chemical structures of the AHLs secreted by A. veronii MTCC 3249.

(a) Compound-1: $N$-tetradecanoylhomoserine lactone; (b) Compound-2: 6-carboxy-HHL.

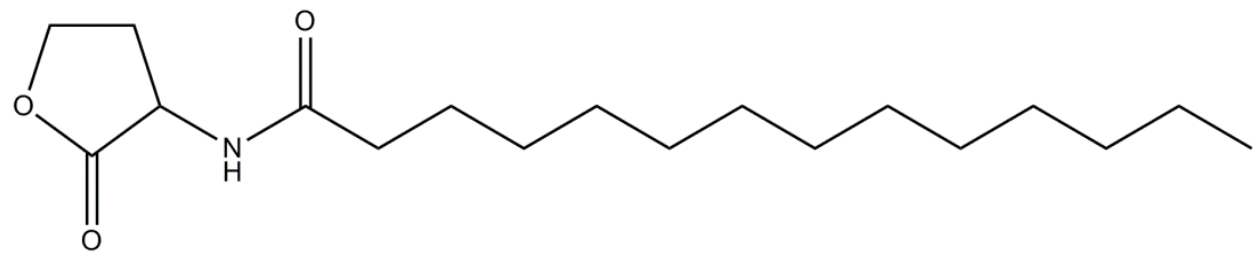

(a)

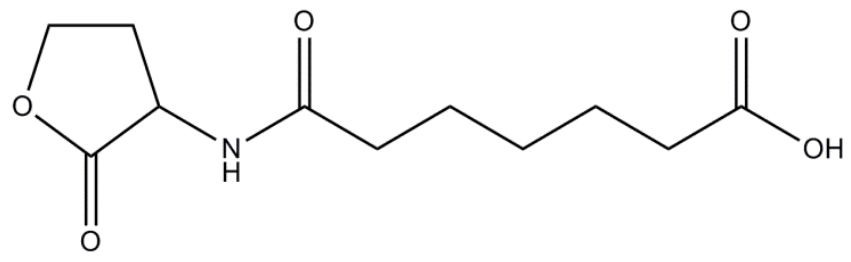

(b)

The results of MS, NMR and IR confirmed that two types of AHL molecules were secreted by A. veronii MTCC 3249 of which one was unique. The production of 6-carboxy-HHL by A. veronii is the first report from any bacterium, the only other being from a methanogenic archaeon, Methanosaeta harundinacea [28]. In addition, A. veronii MTCC 3249 was recently shown to produce several methyl-branched AHLs as well [29]. While the methyl-branched AHLs contained hydroxy acyl chains and unsaturated long acyl chains, our compounds contained neither of these. Although we were unable to detect these methyl-branched AHLs in the HPLC fractions in our study, further chemical analysis of purified fractions from large volumes of culture supernatants is expected to clarify such anomalies.

It is very intriguing that a single strain produces so many different molecules that are potentially under control of a single system. The involvement of such unique types of AHL molecules and the presence of indels in the C-terminal binding domain of AcuR suggests a variant quorum sensing system that is potentially active in A. veronii MTCC 3249 [27]. It is interesting to note that this is the first study that reports the involvement of a carboxyl-AHL derivative in the genus Aeromonas or for any other bacterial genera.

\section{Conclusions}

We propose the presence of a diverse quorum sensing system in A. veronii MTCC 3249 compared to known luxRI homologs in Aeormonas. The presence of indels in the transcription regulator binding domain, a shorter Lux-box, a novel AHL type and a single copy of the quorum sensing system suggest a tight and highly efficient regulation of mechanisms under its control. The nature of these variations might reflect upon the ecological strategies adopted by the bacterium given the unique habitat and the high cell counts inside the midgut of Culex quinquefasciatus. The results presented here reflect a preliminary investigation into the quorum sensing system of A. veronii MTCC 3249. However, in order to gain a better understanding of its ecological strategies and pathogenic potential, a detailed investigation into the mechanisms under the control of quorum sensing system in this strain is proposed. 


\section{Acknowledgments}

The authors thank Paul Williams (Centre for Biomolecular Sciences, University of Nottingham, UK) and Michael Givskov (BioCentrum-DTU, The Technical University of Denmark, Denmark) for providing the biosensor strains used in the study.

\section{References}

1. Janda, J.M.; Abbott, S.L. The genus Aeromonas: Taxonomy, pathogenicity, and infection. Clin. Microbiol. Rev. 2010, 23, 35-73.

2. Swift, S.; Karlyshev, A.V.; Durant, E.L.; Winson, M.K.; Chhabra, S.R.; Williams, P.; Macintyre, S.; Stewart, G.S.A.B. Quorum sensing in Aeromonas hydrophila and Aeromonas salmonicida: Identification of the LuxRI homologues AhyRI and AsaRI and their cognate signal molecules. J. Bacteriol. 1997, 179, 5271-5281.

3. Jangid, K.; Kong, R.; Patole, M.S.; Shouche, Y.S. luxRI homologs are universally present in the genus Aeromonas. BMC Microbiol. 2007, 7, doi:10.1186/1471-2180-7-93.

4. Swift, S.; Lynch, M.J.; Fish, L.; Kirke, D.F.; Tomas, J.M.; Stewart, G.S.; Williams, P. Quorum sensing-dependent regulation and blockade of exoprotease production in Aeromonas hydrophila. Infect. Immun. 1999, 67, 5192-5199.

5. Huys, G.; Cnockaert, M.; Swings, J. Aeromonas culicicola Pidiyar et al. 2002 is a later subjective synonym of Aeromonas veronii Hickman-Brenner et al. 1987. Syst. Appl. Microbiol. 2005, 28, 604-609.

6. Pidiyar, V.J.; Kaznowski, A.; Badrinarayan, N.; Patole, M.S.; Shouche, Y.S. Aeromonas culicicola sp. nov. (MTCC $3249^{\mathrm{T}}$ ), from the midgut of Culex quinquefasciatus. Int. J. Syst. Evol. Microbiol. 2002, 52, 1773-1778.

7. Mourya, D.T.; Gokhale, M.D.; Pidiyar, V.J.; Barde, P.V.; Patole, M.S.; Mishra, A.C.; Shouche, Y.S. Study of the effect of the midgut bacterial flora of Culex quinquefasciatus on the susceptibility of mosquitoes to Japanese encephalitis virus. Acta Virol. 2002, 46, 257-260.

8. Figueras, M.J.; Suarez-Franquet, A.; Chacon, M.R.; Soler, L.; Navarro, M.; Alejandre, C.; Grasa, B.; Martinez-Murcia, A.J.; Guarro, J. First record of the rare species Aeromonas culicicola from a drinking water supply. Appl. Environ. Microbiol. 2005, 71, 538-541.

9. Lynch, M.J.; Swift, S.; Kirke, D.F.; Keevil, C.W.; Dodd, C.E.; Williams, P. The regulation of biofilm development by quorum sensing in Aeromonas hydrophila. Environ. Microbiol. 2002, 4, $18-28$.

10. Zhu, H.; Thuruthyil, S.J.; Willcox, M.D.P. Production of $\mathrm{N}$-acyl homoserine lactones by Gram-negative bacteria isolated from contact lens wearers. Clin. Exp. Ophthalmol. 2001, 29, $150-152$.

11. DeMaio, J.; Pumpuni, C.B.; Kent, M.; Beier, J.C. The midgut bacterial flora of wild Aedes triseriatus, Culex pipiens and Psorophora columbiae mosquitoes. Am. J. Trop. Med. Hyg. 1996, 54, 219-223.

12. Wüster, A.; Babu, M.M. Conservation and evolutionary dynamics of the agr cell-to-cell communication system across Firmicutes. J. Bacteriol. 2008, 190, 743-746. 
13. Cui, Y.; Chatterjee, A.; Hasegawa, H.; Chatterjee, A.K. Erwinia carotovora subspecies produce duplicate variants of ExpR, LuxR homologs that activate $r s m A$ transcription but differ in their interactions with N-acylhomoserine lactone signals. J. Bacteriol. 2006, 188, 4715-4726.

14. Zhang, Z.; Pierson, L.S. A second quorum-sensing system regulates cell surface properties but not Phenazine antibiotic production in Pseudomonas aureofaciens. Appl. Environ. Microbiol. 2001, 67, 4305-4315.

15. McClean, K.H.; Winson, M.K.; Fish, L.; Taylor, A.; Chhabra, S.R.; Camara, M.; Daykin, M.; Lamb, J.H.; Swift, S.; Bycroft, B.W.; Stewart, G.S.A.B.; Williams, P. Quorum sensing and Chromobacterium violaceum: Exploitation of violacein production and inhibition for the detection of N-acylhomoserine lactones. Microbiology 1997, 143, 3703-3711.

16. Winson, M.K.; Swift, S.; Fish, L.; Throup, J.P.; Jørgensen, F.; Chhabra, S.R.; Bycroft, B.W.; Williams, P.; Stewart, G.S.A.B. Construction and analysis of luxCDABE-based plasmid sensors for investigating $\mathrm{N}$-acylhomoserine lactone-mediated quorum sensing. FEMS Microbiol. Lett. 1998, 163, 185-192.

17. Andersen, J.B.; Heydorn, A.; Hentzer, M.; Eberl, L.; Geisenberger, O.; Christensen, B.B.; Molin, S.; Givskov, M. GFP-based N-acylhomoserine-lactone sensor systems for detection of bacterial communication. Appl. Environ. Microbiol. 2001, 67, 575-585.

18. Gram, L.; Christensen, A.B.; Ravn, L.; Molin, S.; Givskov, M. Production of acylated homoserine lactones by psychrotrophic members of the Enterobacteriaceae isolated from foods. Appl. Environ. Microbiol. 1999, 65, 3458-3463.

19. Sambrook, J.; Fritsch, E.F.; Maniatis, T. Molecular Cloning: A Laboratory Manual, 2nd ed.; Cold Spring Harbor Laboratory: New York, NY, USA, 1989.

20. Hanahan, D. Studies on transformation of Escherichia coli with plasmids. J. Mol. Biol. 1983, 166, 557-580.

21. Pidiyar, V.J.; Jangid, K.; Patole, M.S.; Shouche, Y.S. Analysis of 16S-23S intergenic spacer regions and rrn operon copy number of Aeromonas culicicola MTCC 3249 ${ }^{\mathrm{T}}$. DNA Seq. 2003, 14, 183-194.

22. Shaw, P.; Ping, G.; Daly, S.L.; Cha, C.; Cronan, J.E., Jr.; Rinehart, K.L.; Farrand, S.K. Detecting and characterizing acyl-homoserine lactone signal molecules by thin layer chromatography. Proc. Natl. Acad. Sci. USA 1997, 94, 6036-6041.

23. Condon, C.; Liveris, D.; Squires, C.; Schwartz, I.; Squires, C.L. rRNA operon multiplicity in Escherichia coli and the physiological implications of rrn inactivation. J. Bacteriol. 1995, 177, 4152-4156.

24. Stevenson, B.S.; Schmidt, T.M. Growth rate-dependent expression of RNA from plasmid-borne rRNA operons in Escherichia coli. J. Bacteriol. 1997, 180, 1970-1972.

25. Klappenbach, J.A.; Dunbar, J.M.; Schindt, T.M. rRNA operon copy number reflects ecological strategies of bacteria. Appl. Environ. Microbiol. 2000, 66, 1328-1333.

26. Rainey, F.A.; Ward-Rainey, N.L.; Janssen, P.H.; Hippe, H. Clostridium paradoxum DSM 7308(T) contains multiple 16S rRNA genes with heterogeneous intervening sequences. Microbiology 1996, 142, 2087-2095.

27. Jangid, K. Molecular Taxonomy of Genus Aeromonas and Quorum Sensing in Aeromonas culicicola. Ph.D. Thesis, University of Pune, Pune, India, 8 November 2005. 
28. Zhang, G.; Zhang, F.; Ding, G.; Li, J.; Guo, X.; Zhu, J.; Zhou, L.; Cai, S.; Liu, X.; Luo, Y.; Zhang, G.; Shi, W.; Dong, X. Acyl homoserine lactone-based quorum sensing in a methanogenic archaeon. ISME J. 2012, doi:10.1038/ismej.2011.203.

29. Thiel, V.; Kunze, B.; Verma, P.; Wagner-Döbler, I.; Schulz, S. New structural variants of homoserine lactones in Bacteria. ChemBioChem 2009, 10, 1861-1868.

(C) 2012 by the authors; licensee MDPI, Basel, Switzerland. This article is an open access article distributed under the terms and conditions of the Creative Commons Attribution license (http://creativecommons.org/licenses/by/3.0/). 